\title{
Erratum to: Neutron Star Mergers and Gamma-Ray Bursts: Stripping Model
}

\author{
S. I. Blinnikov ${ }^{a, b}, *$, D. K. Nadyozhin ${ }^{a, c, * * \dagger}$, N. I. Kramarev ${ }^{a, d, * * *}$, and A. V. Yudin ${ }^{a, c, * * * *}$ \\ aAlikhanov Institute for Theoretical and Experimental Physics, National Research Center "Kurchatov Institute", \\ Moscow, Russia \\ ${ }^{b}$ Kavli IPMU, Tokyo University, Kashiwa (WPI), Japan \\ ${ }^{c}$ National Research Center "Kurchatov Institute", Moscow, Russia \\ ${ }^{d}$ Moscow State University, Moscow, Russia \\ *e-mail: sblinnikov@gmail.com \\ **e-mail: nadezhin@itep.ru \\ ***e-mail: kramarev-nikita@mail.ru \\ ****e-mail:yudin@itep.ru
}

Received June 21, 2021; revised June 21, 2021; accepted June 21, 2021

DOI: $10.1134 / \mathrm{S} 1063772921340011$

The article "Neutron Star Mergers and GammaRay Bursts: Stripping Model”, written by S.I. Blinnikov, D.K. Nadyozhin, N.I. Kramarev, and A.V. Yudin, was originally published electronically in Springer-Link on 17 May 2021 without Open Access. After publication in volume 65 , issue 5 , pages $385-391$ the authors decided to make the article an Open Access publication. Therefore, the copyright of the article has been changed to (C) The Author(s), 2021 and the article is

\footnotetext{
$\dagger$ Deceased.
}

forthwith distributed under the terms of a Creative Commons Attribution 4.0 International License (http://creativecommons.org/licenses/by/4.0/, CC BY), which permits use, duplication, adaptation, distribution and reproduction of a work in any medium or format, as long as you cite the original author(s) and publication source, provide a link to the Creative Commons license, and indicate if changes were made.

The original article can be found online at https://doi.org/10.1134/S1063772921050012 